\title{
The impact of conversion to International Classification of Diseases, I0th revision (ICD-I0) on an academic ophthalmology practice
}

This article was published in the following Dove Press journal:

Clinical Ophthalmology

\section{Justin B Hellman \\ Michele C Lim \\ Karen $Y$ Leung \\ Cameron M Blount \\ Glenn Yiu}

Department of Ophthalmology \& Vision Sciences, University of California, Davis Medical Center, Sacramento, CA, USA
Correspondence: Glenn Yiu

Department of Ophthalmology \& Vision Sciences, University of California, Davis Health, 4860 Y Street, Suite 2400, Sacramento, CA 958I7, USA

Tel+I 9167346602

Email gyiu@ucdavis.edu
Purpose: To determine the financial and clinical impact of conversion from International Classification of Disease, 9th revision (ICD-9) to ICD-10 coding.

Design: Retrospective, database study.

Materials and methods: Monthly billing and coding data from 44,564 billable patient encounters at an academic ophthalmology practice were analyzed by subspecialty in the 1-year periods before (October 1, 2014, to September 30, 2015) and after (October 1, 2015, to September 30, 2016) conversion from ICD-9 to ICD-10.

Main outcomes and measures: Primary outcome measures were payments per visit, relative value units per visit, number of visits, and percentage of high-level visits; secondary measures were denials due to coding errors, charges denied due to coding errors, and percentage of unspecified codes used as a primary diagnosis code.

Results: Conversion to ICD-10 did not significantly impact payments per visit $(\$ 306.56 \pm \$ 56.50$ vs $\$ 321.43 \pm \$ 38.12, P=0.42)$, relative value units per visit $(7.15 \pm 0.56$ vs $7.13 \pm 0.84, P=0.95)$, mean volume of visits $(1,887.08 \pm 375.02$ vs $1,863.83 \pm 189.81, P=0.71)$, or percentage of high-level visits $(29.7 \% \pm 4.9 \%, 548$ of 1,881 vs $30.0 \% \pm 1.7 \%, 558$ of $1,864, P=0.81)$. For every 100 visits, the number of coding-related denials increased from $0.98 \pm 0.60$ to $1.84 \pm 0.31$ $(P<0.001)$, and denied charges increased from $\$ 307.42 \pm \$ 443.39$ to $\$ 660.86 \pm \$ 239.47(P=0.002)$. The monthly percentage of unspecified codes used increased from $25.8 \% \pm 1.1 \%$ (485 of 1,881) to $35.0 \% \pm 2.3 \%$ (653 of $1,864, P<0.001)$.

Conclusion: The conversion to ICD-10 did not impact overall revenue or clinical volume in this practice setting, but coding-related denials, denied charges, and the use of unspecified codes increased significantly. We expect these denials to increase in the next year in the absence of Medicare's 1-year grace period.

Keywords: ICD-10, ICD-9, ICD International Classification of Diseases, ophthalmology, electronic health records, clinical coding, medical records, reimbursement

\section{Introduction}

The conversion from the International Classification of Diseases, 9th revision, Clinical Modification (ICD-9-CM) to ICD-10-CM was the largest change to health care coding in America in the last 30 years. ${ }^{1}$ While ICD-10 was approved in 1990 and had been utilized in several countries since as early as 1994, its use only became required in the United States on October 1, 2015. The version used in the United States has several significant modifications suggested during an open comment period and via a field test performed by The American Hospital Association and the American Health Information Management Association. ICD-10-CM, as the version is known, added many more 
codes than the original World Health Organization version used prior to adoption in the United States. ${ }^{2,3}$ The Department of Health and Human Services originally planned to instate ICD-10 on October 1, 2011, but its implementation met resistance. The American Medical Association feared that the costs of implementing ICD-10 would be extreme and could even put smaller practices out of business. ${ }^{1}$ In 2009 , the Department of Health and Human Services changed the date to October 1, 2013. ${ }^{4}$ Two additional delays led to the final implementation date of October 1, 2015..$^{5}$

Each ICD-10 code has space for seven characters, an increase from the five available in ICD-9. ${ }^{6}$ The nearly 70,000 diagnostic codes available in ICD-10 provide a much greater level of detail than the 14,500 codes available in ICD-9, and mapping diagnoses from one to the other is often convoluted and rarely straightforward. ${ }^{7}$ Included in the increased details are codes that specify laterality and severity of disease, features that have a substantial effect on coding in ophthalmology. The increased level of detail is projected to lead to an eventual decrease in denied claims. ${ }^{8,9}$ Unfortunately, this decrease in rejected claims is expected to occur after a period of increased rejected claims lasting from 6 months to 3 years as coders adapt to the new system. ${ }^{8,10-12}$ The more detailed information available from ICD-10 codes is also predicted to more easily identify fraud and abuse, give insurance companies more accurate information in order to make coverage decisions, and improve public health monitoring and research. ${ }^{7,10}$ Notably a dually coded database in Canada showed no improvement in the validity of coding with ICD-10 compared to ICD-9. ${ }^{11}$ However, a Swedish study did show continual improvement in the accuracy of recording conditions using ICD-10 over a 5-year period related to a coding learning curve. ${ }^{12}$

The key concern about ICD-10 has always been cost. ${ }^{9,10,13,14}$ The most recent Nachimson Advisors analysis estimated that a small practice ( 3 physicians, 2 administrators) would lose between $\$ 56,639$ and $\$ 226,105$ through additional training, software upgrades, testing, productivity loss, and payment disruption. A medium-sized practice (10 physicians, 1 coder, 6 administrators) was projected to lose $\$ 213,364$ to $\$ 824,735$, and a large practice (100 physicians, 10 coders, and 64 administrators) was projected to lose $\$ 2,017,151$ to $\$ 8,018,364$. The largest portion of these losses was projected to be due to productivity loss and disruptions in payment. These predictions were for physicians in general and not targeted toward any specific specialty. ${ }^{14}$

In this study, we aim to determine the clinical and financial impacts of conversion to ICD-10 on an academic multispecialty ophthalmology practice with a well-established electronic health record (EHR) system. Since the addition of laterality to codes is a significant change and ocular conditions are almost uniformly lateralized, we hypothesized that the conversion may negatively impact eye care providers. We also investigated whether the increasing complexity of coding some common ophthalmologic diagnoses would lead to more frequent use of unspecified codes.

\section{Materials and methods}

We performed a retrospective database study of deidentified billing records at the University of California (UC), Davis Eye Center in Sacramento, CA. The study protocol followed the tenets of the Declaration of Helsinki and was exempted from approval by the university's Institutional Review Board Committee as the work was not considered human subject research as defined by federal regulation 45 CFR 46.102. Our practice consisted of 16 ophthalmologists and included specialists in cornea, glaucoma, neuro-ophthalmology, oculoplastics, pediatrics, and retina. Optometrists, ophthalmology trainees, and part-time volunteer clinical faculty were not included in the analysis. Over the 2 years of the study, the medical staff was unchanged with the exception of the following: 1) a comprehensive ophthalmologist was on sabbatical and worked only part time before June 2015; 2) a neuro-ophthalmologist left the practice in 2015 and was immediately replaced by another neuro-ophthalmologist who saw fewer patients each month; and 3) a retina specialist was hired into the practice 2 months after the beginning of the study period. These three providers were excluded from the analysis to avoid the confounding effect of changes in their clinical volume.

\section{ICD-I0 implementation strategy}

In our practice, a team of ophthalmic coding specialists reviews and analyzes all patient charts after visits are completed and assigns the coding information for medical billing. From July 1, 2014, until the official implementation of ICD-10, the coders utilized both ICD-9 and ICD-10 codes for additional training. The coding staff remained unchanged throughout the course of the study period, and no additional compensation was provided to the coders during the transition period. These figures do not include the cost of software implementation to the EHR system, which took place across the university medical center prior to ICD-10 implementation.

\section{Financial and clinical impacts}

To analyze the changes in clinical revenue and productivity after ICD-10 conversion, we obtained billing summaries from the UC Davis Eye Center from October 1, 2014, to 
Table I Categorization of ICD-9 and ICD- 10 codes

\begin{tabular}{lll}
\hline ICD-9 codes & ICD-I0 codes & Category \\
\hline $360 . X X-363 . X X, 250 . X X$ & $H 30 . X X X-H 36 . X X X, H 43 . X X X-H 44 . X X X$, & Retina \\
$364 . X X, 370 . X X-372 . X X, 54.43,375.15$ & E08.XXX-EI3.XXX & Cornea \\
& $H 10 . X X X-H I I . X X X, H 04.12$, & Glaucoma \\
$365 . X X$ & $H 15 . X X X-H 22 . X X X, B 00.52$ & Cataract \\
$366 . X X$ & $H 40 . X X X-H 42 . X X X, Q 15.0$ & Refractive \\
$367 . X X$ & $H 25 . X X X-H 28 . X X X$ & Oculoplastics \\
$373 . X X-376 . X X$ except 375.I5 & $H 52 . X X X$ & Pediatric/neuro-ophthalmology \\
$368 . X X, 369 . X X, 377 . X X, 378 . X X$ & $H 00 . X X X-H 05 . X X X$ except H04.I2 & \\
& $H 46 . X X X-H 47 . X X X, H 49 . X X X-H 5 I . X X X$, & Perioperative \\
\hline
\end{tabular}

Abbreviation: ICD, International Classification of Diseases.

September 30, 2016, and examined the mean payments per visit, relative value units (RVUs) per visit, number of visits, and percentage of high-level visits for each month during the 12-month periods before and after ICD-10 implementation on October 1, 2015. High-level visits were defined by Current Procedural Terminology codes 99204, 99205, 92004, 99214, 99215, and 92014. Low-level visit codes were defined by Current Procedural Terminology codes 99201-99203, 99243, 92002, 99211-99213, and 92012. We also evaluated the number of coding-related denials and average denied charges per 100 visits, as well as denied charges as a percentage of total charges during the two periods. Coding-related denials were defined as insurance claims that were denied due to an error in coding. Finally, we assessed coder efficiency by comparing the percentage of charges coded within 10 days of a visit before and after ICD-10 implementation.

\section{Coding behavior}

To evaluate the increased complexity of ICD-10 coding as a potential cause of increased insurance claim denials, we identified the top 15 primary ICD-9 codes used between October 1, 2014, and September 30, 2015. We then assessed the corresponding section of ICD-10 to identify all of the possible corresponding codes. To determine if the increased complexity of ICD-10 resulted in more frequent use of unspecified diagnosis codes, we analyzed the total volume of ICD-9 and ICD-10 codes used as primary diagnoses during the 12-month period before and after ICD-10 implementation and compared the proportion of specified and unspecified codes and their distribution among subspecialty categories. We also analyzed which categories of unspecified codes were used most frequently.

ICD-9 codes were categorized based on blocks in Chapter 6 of the 2016 edition of the ICD-9-CM manual. ICD-10 codes were categorized similarly based on blocks in Chapter 7 of the 2016 edition of the ICD-10-CM manual (Table 1).
Unspecified codes were defined as codes with the term "unspecified" in the name where more specific codes exist that could have been used in their place.

\section{Statistical analysis}

We stored and analyzed the data using Microsoft Excel for Mac 2011 with the Real Statistics Resource Pack and Statistical Package for the Social Sciences (SPSS). Normality of all data distributions were confirmed with the Shapiro-Wilk test. If normality held, we utilized two-tailed Student's $t$-tests, and we used a Wilcoxon Rank-Sum Test for variables that did not follow a normal distribution. Data were reported as mean \pm standard deviation. We performed the Benjamini-Hochberg procedure on each group of tests, separated by department, to control false discovery rate with a $q$ value of 0.05 .

\section{Ethics approval and informed consent}

The study protocol followed the tenets of the Declaration of Helsinki and was exempted from approval by the University of California Davis Institutional Review Board Committee.

\section{Results}

When comparing 12 months of data before and after conversion to ICD-10, we noted no significant change in payments per visit ( $\$ 306.56 \pm \$ 56.50$ vs $\$ 321.43 \pm \$ 38.12, P=0.42)$, RVUs per visit ( $7.15 \pm 0.56$ vs $7.13 \pm 0.84, P=0.95)$, mean volume of visits $(1,887.08 \pm 375.02$ vs $1,863.83 \pm 189.81$, $P=0.71)$, or percentage of high-level visits $(29.7 \% \pm 4.9 \%, 548$ of 1,881 per month vs $30.0 \% \pm 1.7 \%, 558$ of 1,864 per month, $P=0.81)$. However, when comparing changes in denial-related metrics before and after conversion to ICD-10, there were nearly twofold increases in coding-related denials per 100 visits ( $0.98 \pm 0.60$ vs $1.84 \pm 0.31 ; P<0.001)$, charges denied per 100 visits ( $\$ 307.42 \pm \$ 443.39$ vs $\$ 660.86 \pm \$ 239.47 ; P=0.002$ ), and percentage of total charges denied $(0.35 \% \pm 0.51 \%$, 
$\$ 5,556.50$ of $\$ 1,581,345$ vs $0.74 \% \pm 0.30 \%, \$ 12,567.33$ of $\$ 1,701,466 ; P=0.002)$.

When analyzing the same data by subspecialties, we found no significant change in payments per visit, RVUs per visit, number of visits, or percentage of high-level visits in any group after ICD-10 conversion, with the exception of comprehensive ophthalmology, which showed a slight increase in percentage of high-level visits (Table 2). All subspecialties

Table 2 Clinical and financial impacts of ICD-10 conversion by subspecialties in ophthalmology

\begin{tabular}{|c|c|c|c|}
\hline Subspecialty & Pre ICD- 10 & Post ICD-I0 & $P$-value \\
\hline \multicolumn{4}{|l|}{ Cornea } \\
\hline Payments/visit & $\$ 231.49 \pm \$ 47.09$ & $\$ 229.65 \pm \$ 44.21$ & 0.93 \\
\hline RVUs/visit & $5.49 \pm 0.70$ & $5.27 \pm 0.99$ & 0.60 \\
\hline Visits & $451.33 \pm 107.14$ & $437.08 \pm 76.33$ & 0.75 \\
\hline Percentage of high-level visits & $19.2 \% \pm 3.2 \%$ & $17.1 \% \pm 3.9 \%$ & 0.18 \\
\hline Coding-related denials per 100 visits & $0.63 \pm 0.75$ & $1.69 \pm 0.6 \mid$ & 0.004 \\
\hline Charges denied per 100 visits & $\$ 240.48 \pm \$ 639.13$ & $\$ 289.78 \pm \$ 143.36$ & 0.002 \\
\hline Percentage of charges denied & $0.42 \% \pm 1.12 \%$ & $0.49 \% \pm 0.32 \%$ & 0.001 \\
\hline \multicolumn{4}{|l|}{ Comprehensive } \\
\hline Payments/visit & $\$ 723.78 \pm \$ 322.73$ & $\$ 800.84 \pm \$ 337.97$ & 0.39 \\
\hline RVUs/visit & $14.77 \pm 4.85$ & $15.88 \pm 3.97$ & 0.27 \\
\hline Visits & $100.17 \pm 26.27$ & $89.83 \pm 24.49$ & 0.41 \\
\hline Percentage of high-level visits & $49.8 \% \pm 6.7 \%$ & $56.7 \% \pm 15.0 \%$ & 0.01 \\
\hline Coding-related denials per 100 visits & $0.79 \pm 0.93$ & $3.80 \pm 2.90$ & 0.001 \\
\hline Charges denied per 100 visits & $\$ 191.78 \pm \$ 364.23$ & $\$ 1,3|0.7| \pm \$ 1,252.38$ & 0.01 \\
\hline Percentage of charges denied & $0.12 \% \pm 0.20 \%$ & $0.74 \% \pm 0.76 \%$ & 0.01 \\
\hline \multicolumn{4}{|l|}{ Glaucoma } \\
\hline Payments/visit & $\$ 250.14 \pm \$ 71.33$ & $\$ 230.45 \pm \$ 31.45$ & 0.33 \\
\hline RVUs/visit & $5.66 \pm 0.74$ & $5.28 \pm 0.60$ & 0.21 \\
\hline Visits & $515.42 \pm 181.92$ & $530.42 \pm 67.78$ & 0.23 \\
\hline Percentage of high-level visits & $14.9 \% \pm 2.9 \%$ & $16.8 \% \pm 2.6 \%$ & 0.08 \\
\hline Coding-related denials per 100 visits & $0.92 \pm 0.6 \mathrm{I}$ & $1.26 \pm 0.66$ & 0.23 \\
\hline Charges denied per 100 visits & $\$ 301.18 \pm \$ 711.09$ & $\$ 644.85 \pm \$ 634.95$ & 0.03 \\
\hline Percentage of charges denied & $0.49 \% \pm 1.18 \%$ & $0.99 \% \pm 0.99 \%$ & 0.03 \\
\hline \multicolumn{4}{|l|}{ Oculoplastics } \\
\hline Payments/visit & $\$ 778.34 \pm \$ 243.99$ & $\$ 873.56 \pm \$ 315.65$ & 0.27 \\
\hline RVUs/visit & $18.40 \pm 6.07$ & $17.48 \pm 4.53$ & 0.70 \\
\hline Visits & $76.08 \pm 19.37$ & $74.33 \pm 15.85$ & 0.72 \\
\hline Percentage of high-level visits & $4.7 \% \pm 3.3 \%$ & $4.8 \% \pm 2.8 \%$ & 0.93 \\
\hline Coding-related denials per 100 visits & $0.59 \pm 0.93$ & $1.33 \pm 1.28$ & 0.17 \\
\hline Charges denied per 100 visits & $\$ 512.62 \pm \$ 975.00$ & $\$ 1,700.31 \pm \$ 2,063.10$ & 0.06 \\
\hline Percentage of charges denied & $0.34 \% \pm 0.68 \%$ & $0.54 \% \pm 0.72 \%$ & 0.20 \\
\hline \multicolumn{4}{|l|}{ Pediatrics } \\
\hline Payments/visit & $\$ 176.53 \pm \$ 55.18$ & $\$ 185.40 \pm \$ 36.04$ & 0.65 \\
\hline RVUs/visit & $4.43 \pm 0.33$ & $4.52 \pm 0.50$ & 0.53 \\
\hline Visits & $329.92 \pm 54.83$ & $326.42 \pm 47.99$ & 0.86 \\
\hline Percentage of high-level visits & $34.3 \% \pm 7.7 \%$ & $38.1 \% \pm 7.7 \%$ & 0.27 \\
\hline Coding-related denials per 100 visits & $1.75 \pm 1.18$ & $2.33 \pm 1.20$ & 0.31 \\
\hline Charges denied per 100 visits & $\$ 416.36 \pm \$ 565.82$ & $\$ 504.72 \pm \$ 446.95$ & 0.25 \\
\hline Percentage of charges denied & $0.84 \% \pm 1.04 \%$ & $0.93 \% \pm 0.77 \%$ & 0.42 \\
\hline \multicolumn{4}{|l|}{ Retina } \\
\hline Payments/visit & $\$ 409.50 \pm \$ 89.89$ & $\$ 472.87 \pm \$ 86.00$ & 0.18 \\
\hline RVUs/visit & $9.64 \pm 1.37$ & $10.21 \pm 2.17$ & 0.73 \\
\hline Visits & $4|4| .7 \pm 9|.7|$ & $405.75 \pm 69.04$ & 0.95 \\
\hline Percentage of high-level visits & $53.2 \% \pm 15.3 \%$ & $52.9 \% \pm 7.6 \%$ & 0.96 \\
\hline Coding-related denials per 100 visits & $0.94 \pm 0.78$ & $2.15 \pm 1.00$ & 0.001 \\
\hline Charges denied per 100 visits & $\$ 309.66 \pm \$ 406.86$ & $\$ 932.40 \pm \$ 772.08$ & 0.02 \\
\hline Percentage of charges denied & $0.24 \% \pm 0.31 \%$ & $0.66 \% \pm 0.55 \%$ & 0.02 \\
\hline
\end{tabular}

Note: Values expressed as monthly mean \pm standard deviation.

Abbreviations: ICD, International Classification of Diseases; RVUs, relative value units. 
experienced an increase in coding-related denials and denied charges, but only higher-volume services including comprehensive, cornea, and retina reached statistical significance (Table 2).

After identifying the top 15 primary visit diagnosis ICD-9 codes used in the 12-month period before ICD-10 implementation, we found a total of 153 possible corresponding ICD-10 codes. There were only 3 one-to-one mappings, where there was only one ICD-10 code that corresponded with the ICD-9 code in question. For 2 of those, exudative and nonexudative senile macular degeneration, the current version of ICD-10 requires much greater specificity than was required during the study period (Table 3 ).

The overall monthly percentage of unspecified codes used increased from $25.8 \% \pm 1.1 \%$ (485 of 1,881) in the ICD-9 period to $35.0 \% \pm 2.3 \%$ (653 of 1,864) after ICD-10 implementation $(P<0.001)$. Significant increases in the percentage

Table 3 Fifteen most commonly used ICD-9 codes and possible corresponding ICD-10 codes

\begin{tabular}{|c|c|c|}
\hline $\begin{array}{l}\text { Most commonly used } \\
\text { ICD-9 codes }\end{array}$ & $\begin{array}{l}\text { Number } \\
\text { of possible } \\
\text { corresponding } \\
\text { ICD- } 10 \text { codes }\end{array}$ & $\begin{array}{l}\text { Possible } \\
\text { corresponding } \\
\text { ICD-I } 0 \text { codes }\end{array}$ \\
\hline $\begin{array}{l}\text { 365. I I: Primary open-angle } \\
\text { glaucoma }\end{array}$ & $5(20)$ & H40.IIXX \\
\hline 365.00: Preglaucoma, unspecified & 28 & $\mathrm{H} 40.0 \mathrm{XX}$ \\
\hline $\begin{array}{l}\text { V67.09: Follow-up examination, } \\
\text { following other surgery }\end{array}$ & I & Z09 \\
\hline $\begin{array}{l}\text { 250.50: Diabetes mellitus with } \\
\text { ophthalmic manifestations, } \\
\text { type II or unspecified, not stated } \\
\text { as uncontrolled }\end{array}$ & $15(56)$ & EII.3XX \\
\hline 365.04: Ocular hypertension & 8 & $\mathrm{H} 40.0 \mathrm{XX}$ \\
\hline $\begin{array}{l}\text { 362.52: Exudative senile macular } \\
\text { degeneration }\end{array}$ & $I(16)$ & $\mathrm{H} 35.32$ \\
\hline $\begin{array}{l}\text { 366.19: Other and combined } \\
\text { forms of senile cataract }\end{array}$ & 10 & $\mathrm{H} 25 . \mathrm{XXX}$ \\
\hline $\begin{array}{l}\text { 375.15: Tear film insufficiency, } \\
\text { unspecified }\end{array}$ & 4 & H04.I2X \\
\hline $\begin{array}{l}\text { 362.51: Nonexudative senile } \\
\text { macular degeneration }\end{array}$ & I (20) & H35.3IXX \\
\hline $\begin{array}{l}\text { 378.35: Accommodative } \\
\text { component in esotropia }\end{array}$ & I & $\mathrm{H} 50.43$ \\
\hline 364.3: Unspecified iridocyclitis & 38 & $\mathrm{H} 20 . \mathrm{XXX}$ \\
\hline 378.00: Esotropia, unspecified & 13 & $\mathrm{H} 50.0 \mathrm{XX}$ \\
\hline 362.56: Macular puckering & 4 & H35.37X \\
\hline $\begin{array}{l}\text { 365. I2: Low tension open-angle } \\
\text { glaucoma }\end{array}$ & 20 & $\mathrm{H} 40.12 \mathrm{XX}$ \\
\hline $\begin{array}{l}\text { 362.53: Cystoid macular } \\
\text { degeneration }\end{array}$ & 4 & H35.35X \\
\hline
\end{tabular}

Notes: Values in parentheses indicate number of possible codes after October I, 2016, which is outside the study period. $X$ indicates the subclassification of the ICD-10 code.

Abbreviation: ICD, International Classification of Diseases. of unspecified codes were seen in glaucoma $(P<0.001)$, other $(P<0.001)$, pediatrics/neuro-ophthalmology $(P<0.001)$, perioperative $(P=0.01)$, oculoplastics $(P=0.003)$, and refractive $(P<0.001)$ diagnoses. Cataract $(P<0.001)$ and cornea $(P<0.001)$ showed significant decreases in percentage of unspecified codes used, and there were no significant changes in retina $(P=0.025)$ diagnoses. The biggest changes were seen in glaucoma and refractive diagnoses (Figure 1). Codes were most often considered unspecified due to lack of laterality, disease type, or stage (Table 4).

There was no overall difference in coder efficiency in the 1-year periods before and after ICD-10 implementation $(P=0.76)$. However, we noted a reduction in coder efficiency during the first 4 months (October 2015 to January 2016) after ICD-10 conversion, which returned to baseline for the remaining 8 months (Figure 2 ).

\section{Discussion}

Our study found no change in financial and clinical productivity for a mid-sized ophthalmology practice after conversion to ICD-10 in terms of total revenue, RVUs, patient volume, and percentage of high-level visits. Although billing claim denials nearly doubled after ICD-10 conversion, the associated financial loss to the practice was only $0.73 \%$ of total charges. Denied charges due to coding errors increased across nearly all subspecialties. A possible explanation for the increase in denials is the increased complexity of ICD-10, which may result in a higher probability of coding errors or an increased use of unspecified codes. From the 15 most common ICD-9 codes used in our practice, we noted a more than 10-fold increase in corresponding ICD-10 codes (Table 3). This increased complexity likely led to the substantial increase in the use of unspecified ICD-10 codes, which occurred across several of the ophthalmic subspecialties.

Our findings contrast with data released by the Centers for Medicare and Medicaid Services (CMS) in 2015 which show that in the fourth quarter, the total number of submitted claims, rejected claims, and denied claims had not changed in the 3 months after switching to ICD-10. ${ }^{15}$ Their findings may be due to the 1-year grace period after ICD-10 implementation during which CMS stated that "Medicare review contractors will not deny physician or other practitioner claims billed under the Part B physician fee schedule through either automated medical review or complex medical record review based solely on the specificity of the ICD-10 diagnosis code as long as the physician/practitioner used a valid code from the right family." 16 Despite this grace period, our study did show an increase in denied payments due to coding errors. 


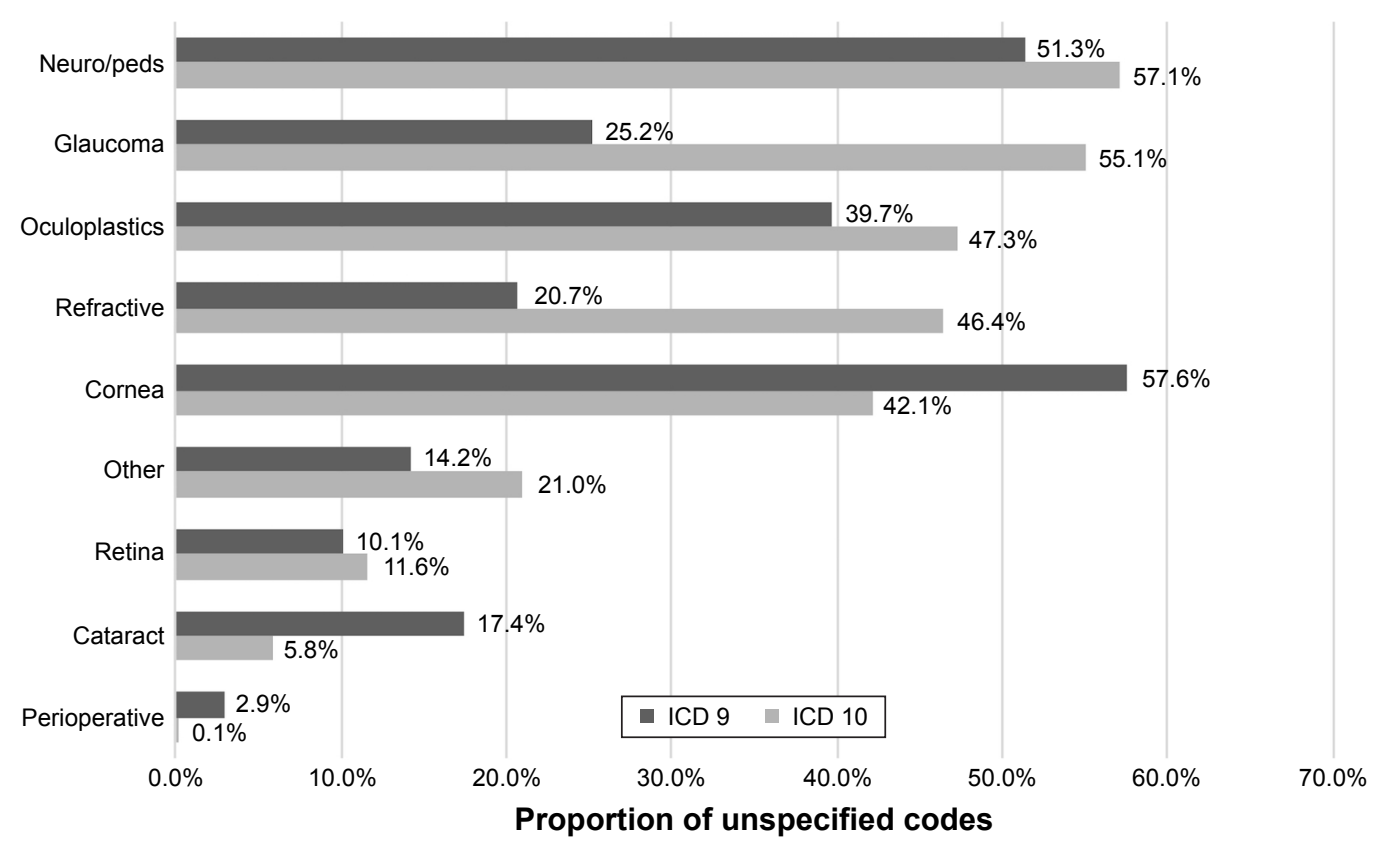

Figure I Proportion of unspecified ICD-9 and ICD-I0 codes at the University of California Davis Eye Center between October I, 20I4, and September 30, 20 I6, categorized by diagnosis type.

Abbreviations: ICD, International Classification of Diseases; Neuro/peds, neuro-ophthalmology and pediatric ophthalmology.

Furthermore, since October 1, 2016, CMS review contractors can use coding specificity as the reason for an audit, and this may lead to a further increase in denied claims.

Nachimson Advisors predicted $\$ 147,912$ to $\$ 501,147$ in productivity loss and payment disruption; however, these predictions do not account for the CMS grace period. With regard to clinical productivity, increased time for documentation and code selection was projected to increase office visit times by $15 \% .{ }^{14}$ Our practice did not experience any significant impact on clinical volume, likely due in part to the employment of a certified coding team in our practice which allows the physicians to spend less time on coding than in other practice settings. Although we noted an early reduction in coder efficiency in the first 4 months after ICD-10 implementation (Figure 2), the overall impact at 1 year was minimal.

A limitation of this study is that we could not calculate the cost of implementing new software and hardware systems for ICD-10 conversion. Nachimson Advisors predicted that a practice of our size would spend $\$ 213,364$ to $\$ 824,735$ in preimplementation costs. ${ }^{14}$ While our practice spent only approximately $\$ 2,000$ for educational materials and coder

Table 4 Most commonly used unspecified ICD-I0 codes and the missing specification

\begin{tabular}{lll}
\hline Unspecified code & $\begin{array}{l}\text { Frequency of use as } \\
\text { primary diagnosis }\end{array}$ & Missing specification \\
\hline Primary open-angle glaucoma, stage unspecified & 998 & Stage \\
Preglaucoma, unspecified, bilateral & 817 & Open or narrow angle \\
Unspecified esotropia & 303 & Monocular or alternating, pattern \\
Unspecified intermittent heterotropia & 286 & Esotropia or exotropia \\
Preglaucoma unspecified, unspecified eye & 279 & Laterality, open or narrow angle \\
Unspecified iridocyclitis & 261 & Primary, recurrent, chronic, or \\
& & secondary, laterality \\
Unspecified corneal scar and opacity & 221 & Central or peripheral, laterality \\
Unspecified glaucoma & 187 & Type, laterality or stage \\
Ocular hypertension, unspecified eye & 154 & Laterality \\
Keratoconus, unspecified, bilateral & 132 & Stable or unstable \\
Unspecified exotropia & 130 & Monocular or alternating, pattern \\
Unspecified amblyopia, right eye & 116 & Type \\
Unspecified amblyopia, left eye & 101 & Type \\
\hline
\end{tabular}

Abbreviation: ICD, International Classification of Diseases. 


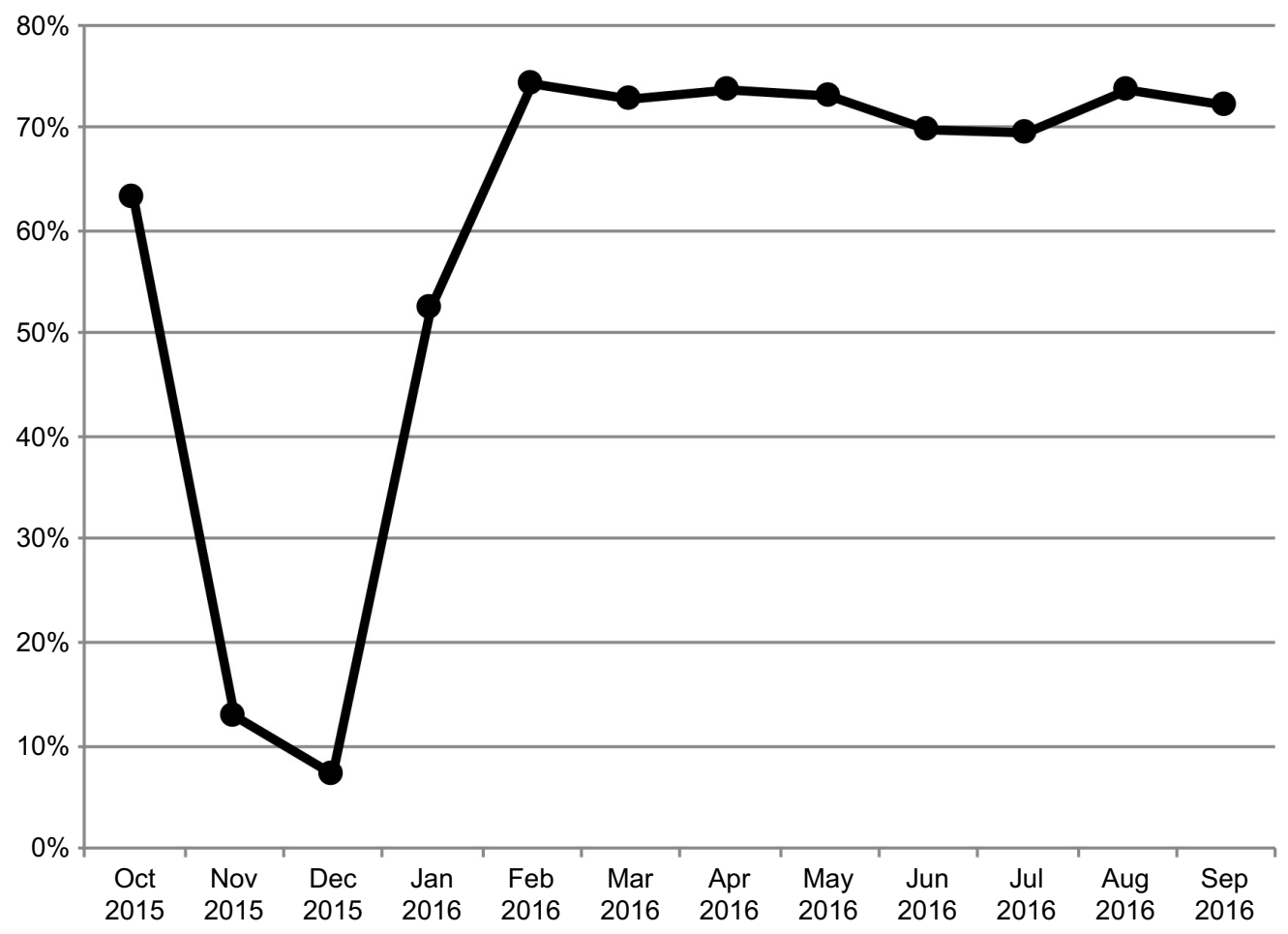

Figure 2 Line graph showing the monthly percentage of transactions coded within 10 days of the visit over the I2-month period following ICD-I0 conversion. Abbreviation: ICD, International Classification of Diseases.

training, the cost of upgrading our EHR system to implement ICD-10 was provided by the UC Davis Health System, and the portion of this cost associated with ophthalmology could not be determined. Another study limitation is that we did not address the use of secondary diagnosis codes or number of codes per patient, and therefore an analysis of the financial impact independent of the ICD-10 codes assigned could not be performed. In addition, since this study compares two different years of practice, it is possible that trends in cases over the study period may have influenced our results. Finally, our study reflects the financial impact of ICD-10 conversion on a medium-sized multispecialty ophthalmology practice in a university setting, and the findings may not readily apply to other practice settings.

Future studies should account for additional changes implemented in ICD-10 for ophthalmology since October 1, 2016. Specifically, open-angle glaucoma and diabetic retinopathy diagnoses now require specification of laterality. Nonexudative age-related macular degeneration requires severity and laterality to be specified. Exudative age-related macular degeneration requires specification of whether there is active choroidal neovascularization, inactive choroidal neovascularization, or an inactive scar. ${ }^{17}$ Finally, retinal vein occlusions require specification of whether macular edema or retinal neovascularization are present. This further increase in specificity may have impacts in the second year after ICD-10 conversion.

\section{Conclusion}

The conversion to ICD-10 has added a much greater level of detail and specificity to coding in ophthalmology. The increase in complexity may have contributed to the increased percentage of unspecified codes used in our practice. It is not clear why we found an increase in coding-related denials and denied charges after conversion to ICD-10 during a grace period in which the use of unspecified codes should not have been penalized by CMS. Nevertheless, ICD-10 implementation did not significantly affect overall financial or clinical productivity over 12 months.

\section{Data availability}

Data is available by request from the corresponding author.

\section{Acknowledgments}

The authors thank Sandra Taylor, principle statistician at the Clinical and Translational Sciences Center at the University of California, Davis, for her help in planning the statistical analysis. Results from this paper were presented in posters at the Academy of Ophthalmology annual meeting in 2016 and at the ARVO annual meeting in 2017. This study 
received no funding. GY is supported by the E. Matilda Ziegler Foundation for the Blind, Barr Foundation for Retinal Research, Alcon Research Institute, ARVO Foundation, California National Primate Research Center, and NIH K08 EY026101.

\section{Disclosure}

GY is a consultant for Allergan, Alimera, Carl Zeiss Meditech, and Iridex. JBH owns stock in Allergan and Ophthotech. For the remaining authors, none were declared. None of these is a competing interest for this study. The authors report no other conflicts of interest in this work.

\section{References}

1. Madara JL (American Medical Association). Letter to: Kathleen Sebelius (US Department of Health and Human Services); 2014. Available from: http://www.asnweb.org/files/Advocacy/2014/ICD\%2010/AMA-letterto-cms-12feb2014.pdf. Accessed February 6, 2018.

2. World Health Organization. International Statistical Classification of Diseases and Related Health Problems: Instruction Manual. 10th revision. Vol. 2. Geneva: WHO; 2011.

3. Rubenstein J. ICD-10: Are you ready? Curr Urol Rep. 2014;15:449.

4. Federal Register/Vol. 74, No 11/Friday, January 16, 2009/Rules and Regulations.

5. Federal Register/Vol. 76, No 235/Wednesday, December 7, 2011/Rules and Regulations.

6. Centers for Medicare and Medicaid Services. ICD-10-CM/PCS: an introduction. Available from: http://www.cms.gov/ICD10/downloads/ ICD-10Overview.pdf. Accessed November 3, 2016.

7. Boyd AD, Li JJ, Burton MD, et al. The discriminatory cost of ICD-10-CM transition between clinical specialties: metrics, case study, and mitigating tools. J Am Med Inform Assoc. 2013;20:708-717.

Clinical Ophthalmology

\section{Publish your work in this journal}

Clinical Ophthalmology is an international, peer-reviewed journal covering all subspecialties within ophthalmology. Key topics include: Optometry; Visual science; Pharmacology and drug therapy in eye diseases; Basic Sciences; Primary and Secondary eye care; Patient Safety and Quality of Care Improvements. This journal is indexed on Submit your manuscript here: http://www.dovepress.com/clinical-ophthalmology-journal
8. Libicki M, Brahmakulam I. The Costs and Benefits of Moving to the ICD-10 Code Sets. Santa Monica, CA: RAND Corporation; 2004.

9. Carr KJ. Closing the ICD-10 revenue gap. Healthc Financ Manage. 2013; 67:118-122.

10. Butler M. Life after ICD-10. How the healthcare world will change after ICD- 10's implementation. J AHIMA. 2015;86:22-27.

11. Quan H, Li B, Saunders LD, et al. Assessing validity of ICD-9-CM and ICD-10 administrative data in recording clinical conditions in a unique dually-coded database. Health Serv Res. 2008;43:1424-1441.

12. Januel JM, Luthi JC, Quan H, et al. Improved accuracy of co-morbidity coding over time after the introduction of ICD-10 administrative data. BMC Health Serv Res. 2011;11:194.

13. Nachimson Advisors, LLC. The impact of implementing ICD-10 on physician practices and clinical laboratories. Available from: http:// www.nachimsonadvisors.com/Documents/ICD-10\%20Impacts $\% 20$ on\%20Providers.pdf. Accessed November 3, 2016.

14. Nachimson Advisors, LLC. The cost of implementing ICD-10 for physician practices - updating the 2008 Nachimson Advisors Study. A report to the American Medical Association; February 12, 2014. Available from: http://docs.house.gov/meetings/IF/IF14/20150211/102940/ HHRG-114-IF14-Wstate-TerryW-20150211-SD001.pdf. Accessed November 3, 2016.

15. CMS [webpage on the Internet]. Lessons learned: reflections on CMS and the successful implementation of ICD-10; 2016. Available from: https:// blog.cms.gov/2016/02/24/lessons-learned-reflections-on-cms-and-thesuccessful-implementation-of-icd-10/. Accessed November 3, 2016.

16. CMS. CMS and AMA announce efforts to help providers get ready for ICD-10. Available from: https://www.cms.gov/Medicare/Coding/ICD10/ Downloads/ICD-10-guidance.pdf. Accessed November 10, 2016.

17. Mcune $\mathrm{D}$ [webpage on the Internet]. ICD-10 updates. An overview of the recent updates for ICD-10, covering coding for wet and dry AMD and glaucoma. Rev Ophthalmol. 2016. Available from: https://www. reviewofophthalmology.com/article/icd10-updates. Accessed November 11, 2016.

\section{Dovepress}

PubMed Central and CAS, and is the official journal of The Society of Clinical Ophthalmology (SCO). The manuscript management system is completely online and includes a very quick and fair peer-review system, which is all easy to use. Visit http://www.dovepress.com/ testimonials.php to read real quotes from published authors. 